\title{
Epileptic seizure: An unusual presentation of takayasu arteritis
}

Background: Takayasu arteritis is a vasculitic disease involving major arteries. It commonly presented with constitutional symptoms. Neurological manifestations such as headache, transient ischemic attack, stroke, vertigo, dementia, confusion, hypertensive encephalopathy and bilateral blindness can be the presenting features rarely. Takayasu arteritis presenting as epileptic seizure is extremely rare. We report a 32 years old previously healthy lady presented with a history of generalized tonic clonic seizure associated with loss of consciousness and ultimately found to have takayasu arteritis and manage with steroids successfully without antiepileptic drugs.

Keywords: takayasu arteritis • epileptic seizure $•$ bruit

\section{Introduction}

Takayasu arteritis is a rare, systemic, inflammatory large-vessel vasculitis of unknown aetiology more common in Asian women. It is defined as granulomatous inflammation of the aorta and its major branches $[1,2]$. It has strong predilection towards aortic arch and proximal part of major branches of aorta. Fever, headache, visual symptoms and constitutional symptoms such as malaise, weight loss are the common mode of presentation. Neurological manifestations may rarely complicate takayasu arteritis. Whereas, seizure as an initial clinical presentation is extremely uncommon.

\section{Case presentation}

A previously healthy 32 years old health care provider lady presented to the emergency department with the history of an episode of generalized tonic clonic seizure with loss of consciousness of 10 minutes duration. She denied any aura symptoms before the episode and there is no bladder or bowel incontinence. A post-ictal drowsiness and a transient impairment of memory were noted. She did not have any history of fever, headache, visual disturbances or limb weakness.

On examination, Glasgow coma scale was 15/15. She was pale without icterus. There was lateral tongue bite mark noted. Detailed neurological examination was normal without any abnormalities in fundus. Cardiovascular examination revealed regular radial pulse rate of 84 beats/min, but there was a mild reduction of pulse volume was noted on right side. Her blood pressure is $140 / 90 \mathrm{mmHg}$ in right arm and $170 / 80 \mathrm{mmHg}$ in the left arm. Both heart sounds were normal and there were no murmurs. Bilateral carotid and vertebral bruits were noted. Bilateral renal bruits were identified on abdominal examination. On targeted history after the clinical examination, patient agreed the intermittent arm pain on the right side.

Hypoglycemia was excluded with normal random blood sugar at presentation. Stroke and space occupying lesions were ruled out with CT brain. She didn't have any electrolyte abnormalities such as sodium, potassium, calcium or magnesium. Her blood investigations revealed, hemoglobin of $6.3 \mathrm{~g} / \mathrm{dl}$ with mean corpuscular volume of $57.8 \mathrm{fl}$ and normal other cell lines. Inflammatory markers were elevated with erythrocyte sedimentation rate of $45 \mathrm{~mm}$ in $1^{\text {st }}$ hour and C-Reactive Protein of $15.5 \mathrm{mg} / \mathrm{L}$. Serum electrolytes, renal function test, liver function test were normal. Electrocardiography, Chest X-ray -PA, electroencephalography and 2D echocardiogram were also normal. Doppler study of Carotid and Vertebral Doppler study was ordered due to the clinical suspicious of

\author{
Sunantha Sriskantharajah*, \\ Peranantharajah Thambipillai \& \\ Nisahan Balasingam \\ Teaching Hospital, Jaffna, Sri Lanka \\ *Author for correspondence: \\ ssuna7@yahoo.co.uk
}


takayasu arteritis and it showed extensive diffuse wall thickening of both common carotid, internal carotid arteries and abdominal aorta. CT aortogram and cerebral vessels angiogram also revealed diffuse arteritis changes mainly involving the thoracic and abdominal aorta, proximal renal vessels, bilateral common carotid arteries, proximal subclavian arteries and brachiocephalic trunk (Figure 1).

According to the American College of Rheumatologist 1990 criteria takayasu arteritis was confirmed as she had five out of six criteria. She was successfully treated with steroids and satisfactory blood pressure control was achieved with amlodipine. Rheumatologist opinion was sought and mycophenolate mofetil was initiated.

\section{Discussion}

Takayasu arteritis (TA) is a rare systemic inflammatory large vessel vasculitis of unknown aetiology, commonly occurs in woman younger than 50 years. Takayasu arteritis can manifest as isolated, atypical or catastrophic disease. It can involve any major organ system. Approximately $10 \%$ of patients are asymptomatic and others mostly presented with headache (50\%-70\%), malaise (35\%-65\%), arthralgia (28\%-75\%), fever (9\%-35\%) and weight loss (10\%-18\%) [3].

Pathogenesis of TA is poorly understood. An autoimmune basis, influenced by genetic and environmental factors is strongly suggested. The resulting inflammation is largely cell-mediated response. The inflammation commences from the adventitia and progresses to the intima and leads to segmental stenosis, occlusion, dilatation and aneurysm formation. Giant cell and granulomatous inflammation are typically found in the media. Progressive inflammation and dense scaring ultimately compromise vascular lumen. Accelerated atherosclerosis may

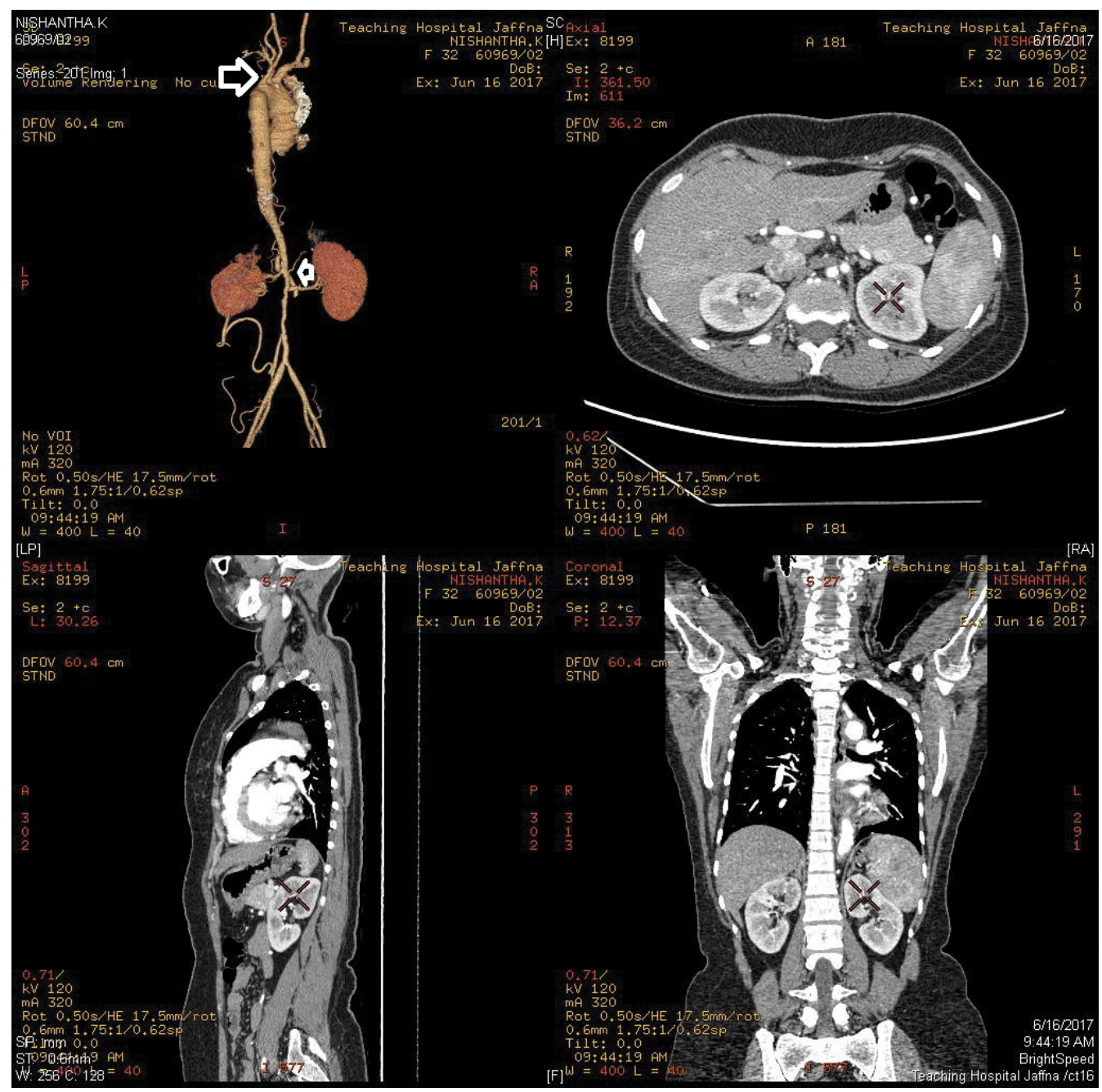

Figure 1. The CT aortogram and cerebral vessels angiogram of $\mathbf{3 2}$ years old lady showing diffuse arteritis changes mainly involving the thoracic and abdominal aorta, proximal renal vessels, bilateral common carotid arteries, proximal subclavian arteries and brachiocephalic trunk. 
also occur in TA [4]. The American college of rheumatology established the diagnosis criteria for takayasu arteritis. The presence of any 3 or more criteria yields a sensitivity of $90.5 \%$ and specificity of $97.8 \%$ [5].

\section{Conclusion}

Takayasu arteritis, a large vessel vasculitis presenting with epileptic seizure is extremely rare. It is associated with substantial morbidity and sometimes may be life-threatening. It is a chronic disease with frequent exacerbations and remissions. There are two major predictors of outcome; one is incidence of complications such as takayasu retinopathy, hypertension, aortic regurgitation and aneurysm, and the other one is presence of a progressive disease [6]. Therefore, we recommend careful detailed clinical examination especially for bruits and blood pressure measurements on both arms is mandatory in young epileptics to avoid catastrophic outcome due to takayasu arteritis.

\section{References}

1. Ioannides MA, Eftychiou C, Georgiou GM et al. Takayasu arteritis presenting as epileptic seizures: a case report and brief review of the literature. Rheumatol. Int. 29(6), 703-5 (2009).

2. Jennette JC, Falk RJ, Bacon PA et al. 2012 revised international chapel hill consensus conference nomenclature of vasculitides. Arthritis. Rheum. 65, $1-11$ (2013).

3. Park MC, Lee SW, Park YB et al. Clinical characteristics and outcomes of Takayasu's arteritis: analysis of 108 patients using standardized criteria for diagnosis, activity assessment, and angiographic classification. Scand. J. Rheumatol. 34(4), 284-92 (2005).

4. Arnaud L, Haroche J, Mathian A et al. Pathogenesis of Takayasu's arteritis: A 2011 update. Autoimmun. Rev. 11, 61-7 (2011).

5. Arend WP, Michel BA, Bloch DA et al. The American College of Rheumatology 1990 criteria for the classification of Takayasu arteritis. Arthritis. Rheum. 33(8), 1129-34 (1990).

6. Phillip R, Luqmani R. Mortality in systemic vasculitis: a systematic review. Clin. Exp. Rheumatol. $26(5$ Suppl 51), S94-104 (2008). 